\section{Recombinant Human Enterovirus 71 in Hand, Foot and Mouth Disease Patients}

\author{
Yoke-Fun Chan* and Sazaly AbuBakar*
}

Two human enterovirus 71 (HEV71) isolates were identified from hand, foot and mouth disease patients with genome sequences that had high similarity to HEV71 $(\geq 93 \%)$ at 5'UTR, P1, and P2 and coxsackievirus A16 (CV$\mathrm{A} 16, \geq 85 \%)$ at $\mathrm{P} 3$ and 3 'UTR. Intertypic recombination is likely to have occurred between HEV71 and CV-A16 or an as-yet to be described CV-A16-like virus.

$\mathrm{H}$ and, foot and mouth disease (HFMD) is a common illness of infants and young children $<10$ years of age. It is characterized by fever, ulcers in the oral cavity, and rashes with blisters that appear on the palm and sole. The most common causal agents of HFMD are coxsackievirus A16 (CV-A16) and human enterovirus 71 (HEV71), but other enteroviruses, including CV-A5 and CV-A10, can also cause it. When caused by CV-A16 infection, it is usually a mild disease, and patients normally recover without requiring any special medical attention.

In rare instances, the disease leads to aseptic meningitis and more serious diseases, such as encephalitis or poliomyelitis-like paralysis. HFMD caused by HEV71 has been associated with the more severe forms of the disease, including a high number of cases of fatal encephalitis during the outbreaks in Malaysia in 1997 and Taiwan in 1998. During these outbreaks, several HEV71 subgenotypes were isolated; two subgenotypes, B4 and $\mathrm{C} 2$, were identified as the main causal agents associated with the fatal infections (1-3). During the Malaysia 1997 outbreak, HEV71 subgenotype B3 was the most prevalent subgenotype isolated from patients with the milder form of HFMD (4). The B3 virus was also found in neighboring countries in 1997 and western Australia in 1999; it has since disappeared (4-6).

To elucidate the mechanisms underlying the emergence of the different HEV71 subgenotypes with potentially different pathogenic potentials, we examined the whole genome sequence of representative HEV71 isolates of HFMD patients from the Malaysia 1997 outbreak. Virus was isolated and identified from patients' samples as

*University of Malaya, Kuala Lumpur, Malaysia described earlier (1). Initial characterization and construction of phylogenetic trees was performed by using the virus genome $5^{\prime}$ nontranslating region (NTR) sequences. From this initial tree, six isolates (UH1 [GenBank accession no. AJ238455], SHA89 [AJ586873], SHA63 [AJ238456], SHA66 [AJ238457], SHA52 [AJ238531], and SHA71 [AJ238535]) were randomly selected to represent the different HEV71 genotypes and were sequenced in their entirety. Sequence analysis, construction of phylogenetic trees, and potential recombination of the isolates were determined as previously described $(1,7)$. Similarity plot and bootscan analyses for the recombination studies were performed with SimPlot version $3.2(8,9)$. For the analysis, a sliding window of $400 \mathrm{nt}$ was moved in increments of $20 \mathrm{nt}$ at a time. Sequences were not corrected for multiple substitutions, all gaps were stripped, transition-totransversion ratio of two was used, and $50 \%$ consensus files were used to exclude the poorly conserved sites. Recombination was identified when conflicting genome sequence profiles appeared, which suggested acquisition of sequences from a different parental genotype. Phylogenetic trees were then constructed for each of the putative recombinant sequences by using the maximum likelihood method, and support for the tree topology was determined by bootscanning analyses that used the bootstrapping procedures (9) with 100 resamplings. The crossover breakpoints were identified when $\chi^{2}$ values were maximum (10). In addition, recombinant sequences were confirmed in patients' samples by using the reverse transcription-polymerase chain reaction (RT-PCR) with primers RECF (5'-CTCAACAGAGCTGTGCTAGTCATGCAATCC-3'; nucleotide positions 5229-5258) and RECR (5'-TCCACTGAGGTTGAGAAAACCATATTGCAC-3'; nucleotide positions 5748-5777), designed on the basis of isolates SHA63 and SHA66 genome sequences and DNA sequencing.

Initially, a phylogenetic tree depicting the genetic relationships of the isolates was constructed by using the whole genome sequence of the six isolates and those available in the GenBank (Figure 1A). As expected, the six isolates were placed into three different lineages (genotypes), and as no reports exist on the typing of the different HEV71 isolates with the whole genome sequences, the genotypes established with the virus capsid (VP1) gene sequence were adopted (5). The three HEV71 subgenotypes identified, B3, B4, and C2, represent the HEV71 genotypes found cocirculating in Malaysia during the 1997 outbreak. Sequence analyses performed by using the isolates whole genome sequences initially reaffirmed the genotyping of the isolates (Figure 1A). The isolates remained within the respective genotypes when the P1 and P2 genome regions were used to construct the phylogenetic trees (online Appendix 1 Figure, available at 


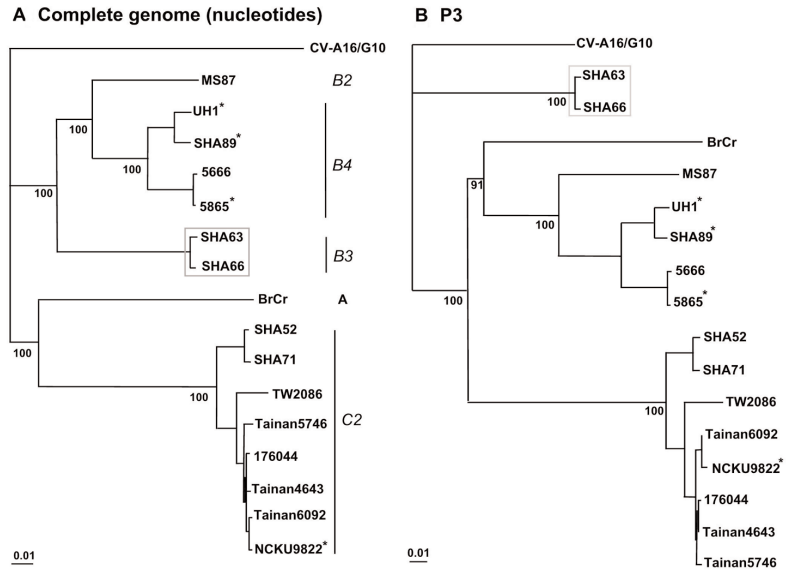

Figure 1. Phylogenetic trees showing genetic relationships among Human Enterovirus 71 (HEV71) isolates. The neighbor-joining trees were constructed from alignment of the whole genome sequences (panel $A$ ) and nucleotide sequences of P3 (nucleotides 5067-7325), (panel B). The bootstrap values are shown as percentage derived from 1,000 samplings, and the scale reflects the number of nucleotide substitutions per site along the branches. Isolates from fatal cases are denoted with asterisks.

http://www.cdc.gov/ncidod/eid/vol10no8/04-0059 app1.htm). A significant shift in the tree topology, which involved the positions of the two subgenotype B3 isolates, SHA63 and SHA66, was noted when the tree was constructed by using the $\mathrm{P} 3$ genome region, a region consisting of the nonstructural protein genes located towards the $3^{\prime}$ end of the genome (Figure 1B). At this genome region, these isolates clustered with the other known causal agent of HFMD, CV-A16. This conflicting tree topology raised the possibility that these isolates contained chimeric genome sequences, perhaps as a result of a previous recombination event involving HEV71 and a human enterovirus A (HEV-A). We performed similarity plot analysis using the consensus genome sequence of HEV71 subgenotype B3 isolates against several potential parental genomes, including all available HEV-A, and confirmed conflicting genome profiles resembling a pattern of recombination for the two subgenotype B3 isolates (Figure 2). The isolates showed high sequence similarity $(\geq 93 \%)$ to subgenotype $\mathrm{B} 4$ at the $5^{\prime}$ terminus of the genome spanning the $5^{\prime}$ NTR region, the whole structural gene sequences, $2 \mathrm{~A}$ and part of 2B gene (nt 1-3908). A conflict in the genome sequence showing high similarity $(\geq 85 \%)$ to $\mathrm{CV}$ A16/G10 was noted at the $3^{\prime}$ end of the genome involving the $3 \mathrm{C}$ protease, $3 \mathrm{D}$ polymerase, and $3^{\prime}$ NTR region gene sequences. Two estimated crossover points that resulted in a switch from HEV71 genotype B to CV-A16-like sequences $(p<0.001$, Fisher exact test) were located within nt 3908-5603. However, the B3 genome sequence dissimilarity relative to subgenotype B2 and B4 viruses began as early as at nt 3617 (part of 2A gene), which does not rule out the possibility that this may be the first crossover point, even though the $\chi^{2}$ values supporting the crossover point was most statistically significant at position 3908 .

Locating a definite breakpoint was not possible within the $\mathrm{B} 3$ virus genome region, as the genome region consisting of part of $2 \mathrm{~B}, 2 \mathrm{C}, 3 \mathrm{~A}, 3 \mathrm{~B}$, and part of $3 \mathrm{C}$ genes had a more complex genome sequence with weak association (low bootstrap values) to all other HEV71 and CV-A16 isolate sequences. Hence, the genome region served as a distinct signature sequence for the subgenotype $\mathrm{B} 3$ isolates.

Specific RT-PCR for detecting CV-A16 VP1 (CV-A16VP1), HEV71 VP1 (HEV71-VP1), and HEV71/CV-A16like sequence (REC) performed on the patients' specimens amplified only enterovirus sequences from all the samples by using the generic enterovirus primer sets that amplified the $5^{\prime}$ NTR (11). This finding suggests that all the samples, except for the controls, contained enterovirus sequences. In contrast, only HEV71-VP1 and HEV71/CV-A16-like

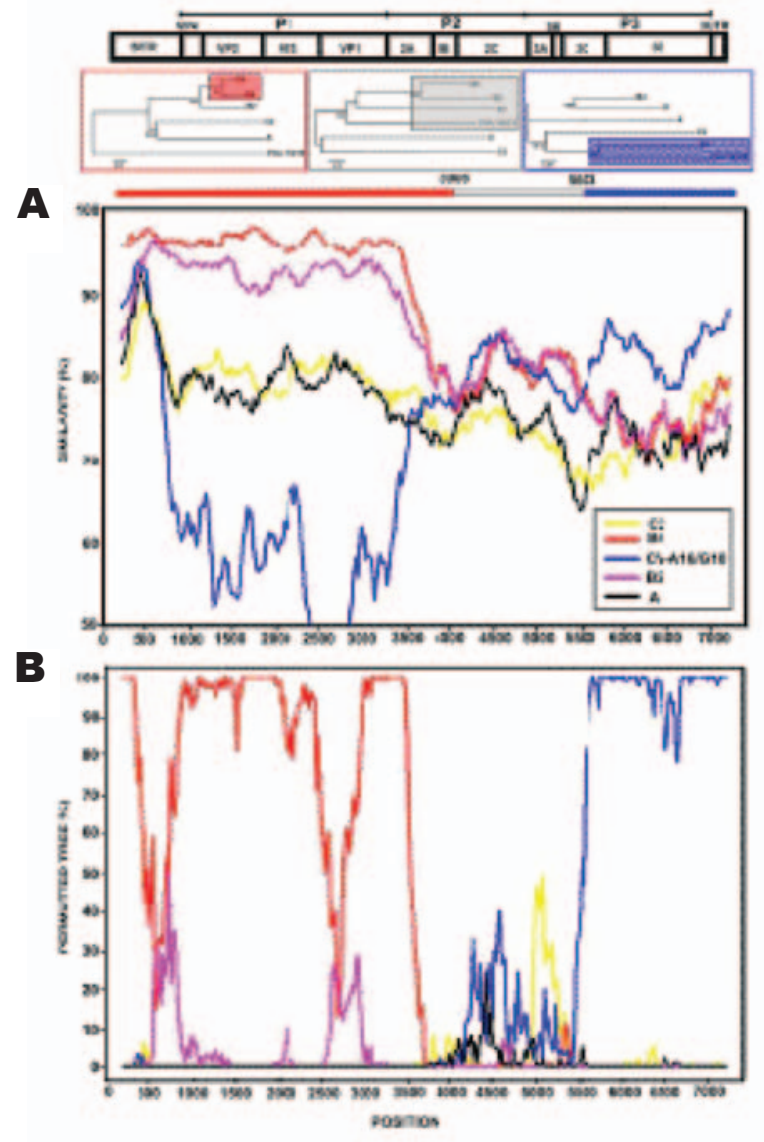

Figure 2. Identification of recombinant sequences in the genome of Human Enterovirus 71 (HEV71) subgenotype B3. A) shows the conflicting tree topology at the P3 genome region. B) shows results from similarity plot and bootscan analyses indicating the recombination sites. The window size of 400-nt slides in increments of 20 nt at a time. Positions containing gaps were excluded from the comparison. 
sequences were amplified from SHA63 and SHA66 samples by using the respective PCR amplification primers (data not shown). No CV-A16-VP1 sequence was detected in either SHA63 or SHA66 patients' samples. These results suggest that the CV-A16-like sequences were not likely to have arisen from a serendipitous sequence amplification artifact involving samples with dual infections with HEV71 and CV-A16. Furthermore, as both of the B3 isolates were obtained from two different HFMD patients (1), the existence of subgenotype B3 HEV71 with CV-A16-like sequences in nature is highly supported.

Further examination of all available 3D polymerase gene sequences (390 nt, subgenotype B3 nucleotide position 6696-7085) of all human enterovirus A (HEV-A) associated with HFMD (12), CV-A5, CV-A10, CV-A16, and HEV71, showed that subgenotype B3 isolates SHA63 and SHA66 had consistently higher nucleotide sequences similarity to CVA-16 (86\%) than to all other HEV71 (75\%-81\%) and HEV-A (76\%-78\%) (online Appendix 2 Table available from http://www.cdc.gov/ncidod/eid/ vol10no8/04-0059_app2.htm). The presence of this CVA16-like sequence within the B3 virus genome suggested that a possible recombination event had previously occurred between HEV71 and CV-A16. The finding that the two viruses tend to cocirculate within the same population during most HFMD outbreaks (13) supported the likelihood that this recombination could have happened. Nonetheless, the parent virus may be a yet-to-be described HEV-A with high sequence similarity to CV-A16.

The CV-A16-like protease and polymerase gene within the B3 isolates genomes could influence the pathogenic potentials of the virus in humans. The B3 virus was likely less pathogenic as the B3 isolates, SHA63, and SHA66, were obtained from uncomplicated HFMD cases, and the B3 virus was not the main virus isolated from children with severe diseases during the outbreaks in Sarawak, Singapore, Malaysia, and Perth (4-6). However, whether all other B3 viruses shared similar characteristics is not known.

Recombination among nonsegmented RNA viruses was once thought to be uncommon. However, findings involving HIV, dengue virus, and poliovirus have established that intratypic recombination does occur among nonsegmented RNA viruses. Similar to enteroviruses (14) and dengue viruses (7), recombination could result in the emergence of viruses with altered pathogenic potentials. In our study, the discovery that two B3 isolates could have emerged as a result of a previous recombination event raises the possibility that recombination events among enteroviruses associated with HFMD occur more frequently in nature. These recombination events could be the mechanism driving the emergence of a number of newly described HEV71 lineages in Asia, some with differing pathogenic potentials.
This study is funded in parts by grants from the Ministry of Science, Technology and Innovation, Malaysia \#06-02-09-001BTK/TD/002.

Ms. Yoke-Fun is currently pursuing a Ph.D. in molecular virology at the University of Malaya. She is a recipient of the National Science Fellowship awarded by the Ministry of Science, Technology and Innovation, Malaysia.

Dr. AbuBakar is a professor of medical microbiology at the University of Malaya. His research interests include the pathogenesis of dengue virus, enterovirus 71 , and Nipah virus infections.

\section{References}

1. AbuBakar S, Chee HY, Al-Kobaisi MF, Xiaoshan J, Chua KB, Lam SK. Identification of enterovirus 71 isolates from an outbreak of hand, foot and mouth disease (HFMD) with fatal cases of encephalomyelitis in Malaysia. Virus Res. 1999;61:1-9.

2. Lum LCS, Wong KT, Lam SK, Chua KB, Goh AYT, Lim WL, et al. Fatal enterovirus 71 encephalomyelitis. J Pediatr. 1998;133:795-8.

3. Wu TN, Tsai SF, Li SF, Lee TF, Huang TM, Wang ML, et al. Sentinel surveillance for Enterovirus 71, Taiwan, 1998. Emerg Infect Dis. 1999;5:458-60.

4. Cardosa MJ, Perera D, Brown BA, Cheon D, Chan HM, Chan KP, et al. Molecular epidemiology of human enterovirus 71 strains and recent outbreaks in the Asia-Pacific region: comparative analysis of the VP1 and VP4 genes. Emerg Infect Dis. 2003;9:461-8.

5. McMinn P, Lindsay K, Perera D, Chan HM, Chan KW, Cardosa MJ. Phylogenetic analysis of enterovirus 71 strains isolated during linked epidemics in Malaysia, Singapore, and Western Australia. J Virol. 2001;75:7732-8.

6. Herrero LJ, Lee CSM, Hurrelbrink RJ, Chua BH, Chua KB, McMinn PC. Molecular epidemiology of enterovirus 71 in peninsular Malaysia, 1997-2000. Arch Virol. 2003;148:1369-85.

7. AbuBakar S, Wong PF, Chan YF. Emergence of dengue virus type 4 genotype IIA in Malaysia. J Gen Virol. 2002;83:2437-42.

8. Lole KS, Bollinger RC, Paranjape RS, Gadkari D, Kulkarni SS, Novak NG, et al. Full-length human immunodeficiency virus type 1 genomes from subtype C-infected seroconverters in India, with evidence of intersubtype recombination. J Virol. 1999;73:152-60.

9. Salminen MO, Carr JK, Burke DS, McCutchan FE. Identification of breakpoints in intergenotypic recombinants of HIV type 1 by bootscanning. AIDS Res Hum Retroviruses. 1995;11:1423-5.

10. Robertson DL, Hahn BH, Sharp PM. Recombination in AIDS viruses. J Mol Evol. 1995;20:249-59.

11. Rotbart HA. Enzymatic RNA amplification of the enteroviruses. J Clin Microbiol. 1990;28:438-42.

12. Pulli T, Koskimies P, Hyypia T. Molecular comparisons of coxsackievirus A virus serotypes. Virology. 1995;212:30-8.

13. Infectious agent surveillance report (IASR). Hand, foot and mouth disease pathogens 1997-2003. [cited 2004 Apr]. Available from: http://idsc.nih.go.jp/prompt/s2graph-k

14. Santti J, Hyypia T, Kinnunen L, Salminen M. Evidence of recombination among enteroviruses. J Virol. 1999;73:8741-9.

Address for correspondence: Sazaly AbuBakar, Department of Medical Microbiology, Faculty of Medicine, University Malaya, 50603 Kuala Lumpur, Malaysia; fax: 60379675757; email: sazaly@um.edu.my 